\title{
ESTRÉS Y ESTRATEGIAS DE AFRONTAMIENTO EN EDUCACIÓN SUPERIOR
}

Amparo Montalvo Prieto

Universidad de Cartagena

Colombia

Miguel Ángel Simancas Pallares

Universidad de Cartagena

Colombia 
Panorama Económico, Vol. 27 - No. 3 (Julio - Septiembre de 2019), pp. 655-674

Amparo Montalvo Prieto

Miguel Ángel Simancas Pallares

\title{
Estrés y estrategias de afrontamiento en educación superior
}

\section{Resumen}

El presente estudio asocia la presencia de estrés académico y las estrategias de afrontamiento utilizadas por los estudiantes universitarios matriculados en una Institución de Educación Superior en Cartagena de Indias (Colombia). Corresponde a un estudio analítico de corte transversal, en el cual participaron 1947 estudiantes matriculados durante el primer período de 2018. Para tal fin se utilizaron tres instrumentos: la Encuesta sobre Aspectos Sociodemográficos, la Escala de Afrontamiento del Estrés Académico (A-CEA) y la Escala de Estrés Académico Percibido (EEAP). Para el análisis bivariado, se empleó el test de Fisher. La comparación de frecuencia de uso de cada una de las estrategias de afrontamiento ante cada situación estresante, se realizó a través de análisis de varianzas multivariante de una vía (MANOVA). Los participantes fueron en su gran mayoría hombres (53.5\%), solteros (95.4\%), de nivel socioeconómico bajo (63.4\%), con pérdida de alguna asignatura (50.23\%). Los estudiantes ante situaciones estresantes utilizan como estrategia de afrontamiento la reevaluación positiva, la planificación y el apoyo social ante la competencia por las calificaciones $(\mathrm{p}=0.00)$; debilidad de carácter $(\mathrm{p}=0.00)$; profesores críticos $(\mathrm{p}=0.00)$ y cantidad de trabajo $(\mathrm{p}=0.02)$. La estrategia de afrontamiento más utilizada por los estudiantes fue la reevaluación positiva ante los diferentes estresores académicos, el establecer los elementos a intervenir y las estrategias a utilizar para fortalecer las respuestas del estudiante ante las situaciones de estrés.

Palabras clave: Estudiante universitario, estrés, reacción

\section{Stress et stratégies de couverture dans l'enseignement supérieur}

\section{Résumé:}

La présente étude associe la présence de stress académique et de stratégies d'adaptation utilisées par les étudiants universitaires inscrits dans un établissement d'enseignement supérieur à Cartagena de Indias (Colombie). Il correspond à une étude analytique transversale à laquelle ont participé 1947 étudiants inscrits au cours de la première période de 2018. Trois instruments ont été utilisés à cette fin: l'enquête sur les aspects sociodémographiques, l'échelle de stress académique (A-CEA) et l'échelle de stress académique perçu (EEAP). Pour l'analyse bivariée, le test de Fisher a été utilisé. La comparaison de la fréquence d'utilisation de chacune des stratégies d'adaptation dans chaque situation stressante a été effectuée par une analyse multivariée de la variance unidirectionnelle (MANOVA). Les participants étaient pour la plupart des hommes (53,5\%), célibataires (95,4\%), de faible statut socio-économique (63,4\%), avec perte d'un sujet $(50,23 \%)$. Les élèves confrontés à des situations stressantes utilisent la réévaluation positive, la planification et le soutien social comme stratégie d'adaptation face à la compétition pour les notes $(p=0,00)$; faiblesse de caractère $(p=0,00)$; Enseignants critiques $(p=0,00)$ et quantité de travail $(p=0,02)$. La stratégie d'adaptation la plus utilisée par les étudiants était la réévaluation positive des différents facteurs de stress académiques, établissant les éléments pour intervenir et les stratégies à utiliser pour renforcer les réponses des étudiants aux situations stressantes.

Mots clés: Etudiant universitaire, stress, réaction

\section{Stress and strategies of coverage in higher education}

\begin{abstract}
The present study associates the presence of academic stress and coping strategies used by university students enrolled in a Higher Education Institution in Cartagena de Indias (Colombia). It corresponds to a cross-sectional analytical study, in which 1947 students enrolled during the first period of 2018 participated. Three instruments were used for this purpose: the Survey on Socio Demographic Aspects, the Academic Stress Coping Scale (A-CEA) and the Perceived Academic Stress Scale (EEAP). For the bivariate analysis, the Fisher test was used. The comparison of the frequency of use of each of the coping strategies in each stressful situation was performed through multivariate analysis of one-way variance (MANOVA). The participants were mostly men (53.5\%), single (95.4\%), of low socioeconomic status (63.4\%), with loss of some subject (50.23\%). Students facing stressful situations use positive reassessment, planning and social support as a coping strategy in the face of competition for grades $(p=0.00)$; weakness of character $(p=0.00)$; Critical teachers $(p=0.00)$ and amount of work $(p=0.02)$. The coping strategy most used by students was the positive reassessment of the different academic stressors, establishing the elements to intervene and the strategies to be used to strengthen the student's responses to stressful situations.
\end{abstract}

Keywords: Out-of-pocket expenses, breast cancer, economic burden. 


\section{Estrés y estrategias de afrontamiento en educación superior}

INFORMACIÓN DEL ARTíCULO

Recepción del artículo: 20/12/2018

Concepto de evaluación: 25/02/2019

Aceptación del artículo: 17/04/2019
Amparo Montalvo Prieto' Universidad de Cartagena, Colombia

Miguel Ángel Simancas Pallares Universidad de Cartagena, Colombia

\section{INTRODUCCIÓN}

El estrés es un proceso que se inicia ante un conjunto de demandas ambientales que recibe el individuo, a las cuales debe dar una respuesta adecuada, poniendo en marcha sus recursos de afrontamiento. Cuando las demandas son excesivas frente a los recursos de afrontamiento que se posee, se va a desarrollar una serie de reacciones adaptativas y movilización de recursos, que implican activación de mecanismos fisiológicos, como son: la ansiedad, la ira y la depresión (SEAS, 2016), síntomas asociados a la enfermedad denominada estrés, considerada un trastorno mental cuando provoca comportamientos sociales desajustados y malestar persistente (Reyes L, 2012).

Una de las etapas de la vida que más produce estrés en las personas es la entrada a la vida universitaria, los jóvenes que ingresan a la educación superior -en edades de 18 a 35 años (Arrieta K, 2013) (Ticona S, 2006) (Gutiérrez J, 2010)- sufren un cambio, debido a que el contexto de la educación secundaria es diferente al universitario y las nuevas exigencias le imponen mayor carga académica (Montalvo A, 2015). Estas nuevas responsabilidades pueden llevar al estudiante a padecer estrés académico, definido por Polo (Polo A, 1996) como aquél que se produce en el espacio educativo e incluye tanto el experimentado por los docentes como por los estudiantes de todos los niveles.

Cuando el estudiante estima que los requerimientos de una situación exceden sus recursos y capacidades, sus pensamientos serán poco esperanzadores y sus emociones negativas, consecuentemente, su esfuerzo y su productividad disminuirán, y como consecuencia puede aparecer el estrés académico (García R \& L, 2012). Esta situación que 
vive el estudiante universitario, interfiere en sus comportamientos adaptativos tales como la dedicación al estudio, la asistencia a las clases o dificultando procesos cognitivos esenciales como la atención y la concentración (Lumley MA, 2003).

(González R, 2010) en un estudio realizado a estudiantes de ciencias de la salud sobre estrés académico, encontró que los exámenes y las intervenciones en público, son las dos situaciones académicas que los estudiantes perciben como más estresantes, seguido por la sobrecarga y las deficiencias metodológicas del profesorado (González R, 2010), así mismo (García R \& L, 2012), encontró que los estudiantes experimentan elevados niveles de estrés ante la falta de tiempo para cumplir con las actividades académicas.

Para hacer frente a esta situación de estrés, el ser humano adopta una serie de estrategias, denominadas afrontamiento, el cual inicia con una evaluación cognitiva que conduce a modificar la situación, aceptar, buscar información, demorar la respuesta, etc. Seconstituye así el afrontamiento en los esfuerzos constantes cognitivos y comportamentales del individuo para gestionar las demandas concretas externas y/o internas que se perciben como agotadoras o excesivas, generar recursos propios, independientemente de si estos esfuerzos sean exitosos o no (Lazarus RS, 1984). El afrontamiento es un proceso que se dirige a lo que la persona realmente piensa y hace en situaciones estresantes, y está influido por las valoraciones de los individuos en torno a las demandas reales a las que se enfrentan y los recursos de los que disponen para abordarlas (Cabanach R, 2010).

Rost (2008) reporta que con frecuencia los estudiantes para resolver los problemas académicos utilizan estrategias de planificación, concentración, supresión de actividades distractoras, aceptación y mantenimiento del optimismo, las cuales pueden considerarse como efectivas y adaptativas (Rost D, 2008), para Piemontesi (2012) el uso frecuente de críticas negativas hacia ellos mismos, con una visión pesimista, pensamientos recurrentes sobre el problema pero que no están orientados a resolverlo, y que en realidad retroalimentan el estado de malestar que experimentan son estilos de afrontamiento que pueden interferir en el estudio y ante la presentación de un examen presentan mayor ansiedad (Piemontesi S, 2012).

Algunas conductas de afrontamiento ante el estrés lo constituyen la búsqueda de apoyo social. Ésta puede presentar una finalidad destinada al control de las emociones (encontrar apoyo afectivo, consuelo), o puede también perseguir un objetivo de tipo instrumental, dirigido a la resolución del problema (obtener información valiosa, un consejo o directamente ayuda). La utilidad de una determinada estrategia de afrontamiento depende, en gran parte, de la naturaleza del estímulo estresor (Fernández M, 2009).

Se desconoce en los estudiantes la intensidad de estrés que presentan y cuáles son los recursos de afrontamiento utilizados para abordar estas situaciones. Partiendo de este contexto se planteó asociar la presencia de estrés académico y las estrategias de afrontamiento utilizadas por los estudiantes matriculados en los diferentes programas en la modalidad presencial de una Institución Universitaria de la ciudad de Cartagena en el año 2018. Esta información es necesaria para establecer y desarrollar programas de intervención, orientados a fortalecer los mecanismos positivos de afrontamiento. 


\section{MATERIALES Y MÉTODOS}

Estudio analítico de corte transversal realizado en una institución universitaria de Cartagena- Colombia, participaron 2017 estudiantes matriculados durante el primer período de 2018. Se solicitó autorización a la institución universitaria, la Oficina de Sistemas proporcionó el listado de los estudiantes matriculados en cada uno de los programas académicos de la modalidad presencial. Para seleccionar la muestra, se definieron aspectos relacionados con cada unidad académica, se calculó teniendo en cuenta una prevalencia esperada del fenómeno 45,4\%, confiabilidad de 95\%, error tipo I 0,05, poder del estudio 80\% y error de muestreo anticipado de 15 \% (García J, 2013). Se empleó una función para estimación puntual de la prevalencia empleando el paquete Tamaño de la Muestra v.1.1.

\section{Tabla 1.}

Población estudiantil matriculada en la modalidad presencial en una institución universitaria. Cartagena, 2018.

\begin{tabular}{|c|c|c|c|}
\hline Facultad & Programa & $\mathbf{N}$ & n \\
\hline \multirow[t]{6}{*}{ Ingenierías } & Alimentos & 366 & 76 \\
\hline & Civil & 516 & 81 \\
\hline & Sistemas & 336 & 75 \\
\hline & Química & 476 & 80 \\
\hline & Sistemas Nocturno & 25 & 20 \\
\hline & $\begin{array}{l}\text { Técnico Profesional en } \\
\text { Operación de Procesos } \\
\text { Petroquímicos }\end{array}$ & 57 & 36 \\
\hline \multirow[t]{7}{*}{ Ciencias Económicas } & Administración de & & \\
\hline & Empresas Nocturno & 456 & 79 \\
\hline & Economía & 369 & 76 \\
\hline & $\begin{array}{l}\text { Administración de } \\
\text { Empresas Diurno }\end{array}$ & 502 & 81 \\
\hline & Contaduría Nocturno & 441 & 79 \\
\hline & Administración Industrial & 498 & 81 \\
\hline & Contaduría Diurno & 500 & 81 \\
\hline \multirow{5}{*}{$\begin{array}{l}\text { Ciencias Exactas } \\
\text { y Naturales }\end{array}$} & Matemáticas & 154 & 56 \\
\hline & Química & 204 & 59 \\
\hline & Biología & 133 & 66 \\
\hline & $\begin{array}{l}\text { Técnica Profesional en } \\
\text { Procesos Metrológicos }\end{array}$ & 57 & 36 \\
\hline & $\begin{array}{l}\text { Tecnología en Metrología } \\
\text { Industrial }\end{array}$ & 27 & 21 \\
\hline
\end{tabular}


Tabla 1.

Población estudiantil matriculada en la modalidad presencial en una institución universitaria. Cartagena, 2018.

\begin{tabular}{|c|c|c|c|}
\hline Facultad & Programa & $\mathbf{N}$ & $\mathrm{n}$ \\
\hline \multirow[t]{4}{*}{ Ciencias Humanas } & Historia & 157 & 60 \\
\hline & Filosofía & 122 & 54 \\
\hline & Lingüística y Literatura & 239 & 69 \\
\hline & $\begin{array}{l}\text { Lenguas Extranjeras con } \\
\text { énfasis en Inglés y Francés }\end{array}$ & 239 & 69 \\
\hline \multirow{3}{*}{$\begin{array}{l}\text { Ciencias Sociales } \\
\text { y Educación }\end{array}$} & Comunicación Social & 506 & 81 \\
\hline & Trabajo Social & 431 & 79 \\
\hline & $\begin{array}{l}\text { Licenciatura en Educación } \\
\text { con Énfasis en Ciencias } \\
\text { Sociales y Ambientales }\end{array}$ & 152 & 59 \\
\hline Ciencias Farmacéuticas & Química Farmacéutica & 327 & 74 \\
\hline \multirow{2}{*}{$\begin{array}{l}\text { Derecho y } \\
\text { Ciencias Políticas }\end{array}$} & Derecho & 770 & 85 \\
\hline & Derecho vespertino & 146 & 58 \\
\hline Enfermería & Enfermería & 488 & 80 \\
\hline Odontología & Odontología & 496 & 81 \\
\hline \multirow[t]{2}{*}{ Medicina } & Medicina & 761 & 85 \\
\hline & TOTAL & 9.960 & 2.017 \\
\hline
\end{tabular}

Fuente: Registro académico Oficina de Admisiones. 
De la muestra seleccionada (2017 estudiantes) (Tabla 1), se estimó un cronograma para la aplicación del instrumento a partir de la ubicación en un solo intento al grupo para obtener los datos. Se utilizaron tres instrumentos, la encuesta sobre aspectos sociodemográficos, la escala de afrontamiento del estrés académico (A-CEA) propuesta por Cabanach (Cabanach R, 2010) (Sandín B, 2003), con respuestas tipo Likert con cinco opciones de respuesta donde 1 corresponde a nunca, 2 alguna vez, 3 bastantes veces, 4 muchas veces y 5 Siempre. Consta de 23 ítems y tres factores; el primero denominado Reevaluación Positiva, constituido por 9 ítems (1, 4, 7, 10,13, 16, 18, 20 y 22), se refiere a una forma de afrontamiento activo dirigida a crear un nuevo significado de la situación. El segundo denominado Búsqueda de Apoyo Social constituido por 7 ítems (2, 5, 8, 11, 14, 17 y 21) y contempla la ayuda para la comprensión de la situación emocional causada por el problema, el tercer factor lo conforman 7 ítems (3, 6, 9, 12, 15, 19 y 23) y es designado planificación, referidos a estrategias de afrontamiento dirigidas a modificar o controlar instrumentalmente la situación. La escala presentó un alpha de Cronbach de 0,893 para el total de los 23 ítems que la componen, 0,864 para el factor Reevaluación Positiva; de 0,906 para el factor Búsqueda de Apoyo, y 0,837 para el factor Planificación. En el presente estudio la consistencia interna obtenida fue de 0,91. El tercer instrumento fue la escala de Estrés Académico Percibido (EEAP) elaborada por (Bedewy D, 2015), autorizada su utilización a través del pago de los derechos de autor, consta de 18 items, con cinco opciones de respuesta en una escala tipo likert, donde 1 es completamente en desacuerdo, 2 en desacuerdo, 3 ni en desacuerdo ni de acuerdo, 4 de acuerdo y 5 completamente de acuerdo. La consistencia interna de la EEAP fue de 0.71, está constituida por cuatro dimensiones que corresponden a presiones para el desempeño, consta de cinco ítems (1-5), el factor percepciones de la carga académica contiene cuatro items (6-9), autopercepciones académicas con cuatro items (10-13) y con cinco ítems el factor limitaciones de tiempo (14-18) (22).

Como el instrumento de (Bedewy D, 2015) sólo se encuentra disponible en inglés, fue necesario adaptarlo al español, siguiendo las recomendaciones metodológicas propuestas por Beaton y Bombardier (Beaton DE, 2000) en 6 fases: inicialmente se realizaron dos traducciones independientes de inglés a español (fase I), se sintetizaron las versiones obtenidas (fase I), se resolvieron discrepancias entre los traductores (fase II). En la fase III o de traducción reversa, se obtuvieron dos traducciones al inglés del cuestionario propuesto previamente en español. En la fase IV se revisaron y se generó una versión pre-final en español del instrumento. En la fase V se realizó una prueba piloto con 40 estudiantes (no hicieron parte de la muestra) para probar el entendimiento de cada ítem y determinar mínimos ajustes de gramática, cohesión, etc. Finalmente, en la fase VI se generó una versión final que fue la empleada en el presente estudio.

Para recolectar la información participaron auxiliares de investigación, quienes fueron capacitados en el manejo de los instrumentos y en la selección de los estudiantes. Los Jefes de departamento académico de cada programa autorizaron la entrada para la recolección de la información; al aceptar participar en el estudio, los estudiantes firmaron el consentimiento informado y diligenciaron los instrumentos en presencia del auxiliar de investigación.

Fueron rechazadas 70 encuestas por presentar información incompleta. Los datos fueron digitados una tabla matriz en Microsoft Excel.

Inicialmente se verificó la normalidad de la distribución de los datos continuos usando la prueba Shapiro-Wilks. En la fase descriptiva del análisis se calcularon frecuencias y 
proporciones para variables cualitativas y dependiendo de la distribución de los datos media y desviación estándar o mediana y rango intercuartílico. Adicionalmente, se calculó estadística descriptiva para cada uno de los ítems y dominios de las escalas de medición. La consistencia interna para cada escala se evaluó empleando el coeficiente alfa de Cronbach.

En la escala de estrés académico percibido cada ítem tiene respuestas que oscilan entre 1 y 5 . Con el fin de permitir una mayor interpretación a las estrategias de afrontamiento empleadas para cada estresor, se creó para cada ítem una respuesta dicotómica colapsando las opciones de respuesta así: presencia de estrés/estresor (opciones 3, 4 y 5) y ausencia de estrés/estresor (opciones 1 y 2). Aun cuando esta escala sugiere agrupar ítems de acuerdo a dominios, se decidió evaluar esta asociación para cada ítem dado que cada uno evalúa una situación diferente. De otro lado, se concibió teóricamente una estrategia de afrontamiento a cada uno de los dominios de la escala de afrontamiento del estrés académico (reevaluación positiva, búsqueda de apoyo y planificación).

Para el análisis bivariado, se empleó el test exacto de Fisher (en caso que haya valores esperados $\leq 5$ ). En el análisis multivariado, se obtuvo un modelo empleando regresión lineal definiendo como variable dependiente al puntaje de la escala CAE. Los estimadores de asociación de esta regresión, serán expresados como betas e intervalos de confianza al 95 \%. Asimismo, estos estimadores serán ajustados por sexo y edad. Para la estimación de las propiedades psicométricas de cada instrumento, se realizó: análisis de consistencia interna con coeficiente alfa de Cronbach y fórmula 20 de Kuder-Richardson, validez de constructo con análisis factorial exploratorio y confirmatorio. El análisis de los datos se realizó en el paquete estadístico STATA ${ }^{\mathrm{TM}}$ v.12.3 para Windows. El análisis factorial confirmatorio se realizará empleando Mplus v.7.31 para Windows. Finalmente, se asumirá significancia estadística cuando $\mathrm{p} \leq 0.05$.

La comparación de los promedios de las posibles estrategias de afrontamiento (A-CEA) de acuerdo a la presencia/ausencia de cada estresor, se realizó a través de análisis de varianzas multivariante de una vía o MANOVA. Todos los análisis se realizaron empleando Stata v.15.1 para Windows (StataCorp., TX, US).

\section{RESULTADOS}

La muestra final estuvo constituida por 1947 estudiantes, en su gran mayoría hombres (53.5\%), solteros (95.4\%), de nivel socioeconómico bajo (63.4\%), con pérdida de alguna asignatura (50.23\%), profesan creencia religiosa católica (54.96\%) y pertenecen a familia nuclear (62.6\%). Sólo una pequeña proporción de los encuestados actualmente trabaja (14.64\%) y la mitad están afiliados al régimen subsidiado de salud (50.49\%). La edad promedio global fue $24.9 \pm 3.0$ años (Tabla 2 ). 
Tabla 2.

Características sociodemográficas de los estudiantes de una institución universitaria, Cartagena, 2018.

\begin{tabular}{|c|c|c|}
\hline Característica & $\mathrm{n}$ & Prop \\
\hline \multicolumn{3}{|l|}{ Sexo } \\
\hline Femenino & 904 & 46.43 \\
\hline Masculino & 1043 & 53.57 \\
\hline \multicolumn{3}{|l|}{ Estado Civil } \\
\hline Soltero & 1859 & 95.48 \\
\hline Casado & 21 & 1.08 \\
\hline Unión Libre & 55 & 2.82 \\
\hline Separado/Divorciado & 7 & 0.36 \\
\hline Viudo(a) & 5 & 0.26 \\
\hline \multicolumn{3}{|l|}{ Nivel Socioeconómico } \\
\hline Bajo & 1235 & 63.43 \\
\hline Medio & 660 & 33.9 \\
\hline Alto & 52 & 2.67 \\
\hline \multicolumn{3}{|l|}{ Facultad } \\
\hline Ingeniería & 339 & 17.41 \\
\hline Ciencias Económicas & 465 & 23.88 \\
\hline Ciencias Naturales & 261 & 13.41 \\
\hline Ciencias Humanas & 215 & 11.04 \\
\hline Ciencias Sociales & 207 & 10.63 \\
\hline Ciencias Farmacéuticas & 68 & 3.49 \\
\hline Derecho & 143 & 7.34 \\
\hline Enfermería & 79 & 4.06 \\
\hline Medicina & 82 & 4.21 \\
\hline Odontología & 88 & 4.52 \\
\hline \multicolumn{3}{|c|}{ ¿Ha perdido alguna asignatura? } \\
\hline No & 969 & 49.77 \\
\hline Sí & 978 & 50.23 \\
\hline
\end{tabular}


Tabla 2.

Características sociodemográficas de los estudiantes de una institución universitaria, Cartagena, 2018.

\begin{tabular}{|c|c|c|}
\hline Característica & $\mathrm{n}$ & Prop \\
\hline \multicolumn{3}{|c|}{ Creencia religiosa } \\
\hline Católico & 1070 & 54.96 \\
\hline Evangélico & 257 & 13.2 \\
\hline Otra & 453 & 23.27 \\
\hline Ninguna & 167 & 8.58 \\
\hline \multicolumn{3}{|l|}{ Tipo de Familia } \\
\hline Nuclear & 1219 & 62.61 \\
\hline Monoparental & 224 & 11.5 \\
\hline Extensa & 96 & 4.93 \\
\hline Otra & 408 & 20.96 \\
\hline \multicolumn{3}{|c|}{ Trabaja Actualmente } \\
\hline No & 1662 & 85.36 \\
\hline Sí & 285 & 14.64 \\
\hline \multicolumn{3}{|c|}{ Régimen de afiliación al SGSSS } \\
\hline Especial & 41 & 2.11 \\
\hline Contributivo & 903 & 46.38 \\
\hline Subsidiado & 983 & 50.49 \\
\hline Sin afiliación & 20 & 1.03 \\
\hline
\end{tabular}

De la Facultad de Ciencias Económicas participaron 465 estudiantes, $57.8 \%$ de sexo masculino, en su mayoría solteros. El 53\% ha perdido asignaturas, de ellos el $23.2 \%$ sólo una y el $5.6 \%$ más de cuatro asignaturas.

La EEAP alcanzó una media global de 3.09ะ0.48 sugiriendo que en su gran mayoría los estudiantes reportaron estar "ni en desacuerdo, ni de acuerdo" con cada uno de los estresores interrogados. Los dominios presiones para el desempeño, percepciones de la carga académica, auto-percepciones académicas y limitaciones de tiempo alcanzaron promedios de $2.87 \pm 0.77,3.09 \pm 0.80,3.54 \pm 0.67$ y $2.82 \pm 0.64$, respectivamente. El análisis estadístico de los datos reporta que los estudiantes perciben en orden secuencial 
principalmente los siguientes estresores, estoy seguro que seré exitoso en mi futura carrera, estoy seguro que seré un estudiante exitoso (auto-percepción académica) y el período de exámenes es muy estresante (presiones para el desempeño), las preguntas de los exámenes (percepción de la carga académica). A su vez percibieron como menos estresantes los estresores, las expectativas de los profesores, el no ponerse al día en sus actividades (limitaciones de tiempo) y las expectativas de los padres (presiones para el desempeño) (Tabla 3).

Tabla 3.

Estadística descriptiva para cada uno de los ítems de la escala de estrés académico percibido (EEAP), en estudiantes de una institución universitaria, Cartagena, 2018.

\begin{tabular}{|c|c|c|c|c|c|c|c|c|}
\hline \multirow[b]{2}{*}{ Item } & \multirow[b]{2}{*}{ Media } & \multirow[b]{2}{*}{$\mathrm{DE}$} & \multirow[b]{2}{*}{ Ranking } & \multicolumn{5}{|c|}{ Proporción de Respuesta (\%) } \\
\hline & & & & 1 & 2 & 3 & 4 & 5 \\
\hline Éxito en el futuro & 3.98 & 1.09 & 1 & 3 & 7 & 19 & 29 & 41 \\
\hline Estudiante exitoso(a) & 3.86 & 1.03 & 2 & 3 & 7 & 22 & 36 & 31 \\
\hline Período de exámenes & 3.56 & 1.21 & 3 & 7 & 13 & 23 & 30 & 27 \\
\hline Reprobar asignaturas & 3.44 & 1.32 & 4 & 11 & 15 & 21 & 25 & 28 \\
\hline Decisiones académicas & 3.44 & 1.05 & 5 & 5 & 13 & 30 & 37 & 15 \\
\hline Preguntas de los exámenes & 3.26 & 1.04 & 6 & 6 & 15 & 38 & 30 & 12 \\
\hline Conseguir trabajo & 3.20 & 1.30 & 7 & 14 & 18 & 24 & 27 & 19 \\
\hline Tiempo académico & 3.15 & 1.09 & 8 & 9 & 18 & 33 & 31 & 9 \\
\hline Tiempo para relajarme & 3.01 & 1.22 & 9 & 14 & 21 & 28 & 25 & 12 \\
\hline Cantidad de trabajo & 3.00 & 1.09 & 10 & 10 & 21 & 39 & 21 & 10 \\
\hline Tiempo de los exámenes & 2.98 & 1.16 & 11 & 13 & 20 & 34 & 23 & 10 \\
\hline Profesores críticos & 2.97 & 1.11 & 12 & 12 & 19 & 36 & 25 & 8 \\
\hline Tamaño del plan de estudios & 2.90 & 1.09 & 13 & 11 & 25 & 34 & 22 & 8 \\
\hline Competencia por calificaciones & 2.83 & 1.13 & 14 & 15 & 22 & 34 & 22 & 7 \\
\hline Debilidad de carácter & 2.67 & 1.18 & 15 & 19 & 27 & 29 & 17 & 8 \\
\hline Expectativas de Profesores & 2.57 & 1.11 & 16 & 22 & 22 & 39 & 12 & 5 \\
\hline Incapaz de ponerme al día & 2.43 & 1.23 & 17 & 29 & 28 & 21 & 15 & 7 \\
\hline Expectativas de los Padres & 2.41 & 1.23 & 18 & 30 & 26 & 23 & 14 & 7 \\
\hline
\end{tabular}

El 32,9\% de los estudiantes de la Facultad de Ciencias Económicas manifestaron que el período de los exámenes (parciales) es muy estresante para y para el 20,9\% la competencia con sus compañeros por las calificaciones es bastante intensa. En cuanto a la evaluación del rendimiento por parte de los docentes, el 23,9\% consideró que los profesores son críticos de su rendimiento académico, pero el $40 \%$ se consideran estudiantes exitosos y el $28 \%$ teme reprobar asignaturas en el semestre. 
Con relación a la A-CEA alcanzó una media global de 3.03×0.66 sugiriendo queen promedio los estudiantes emplearon "bastantes veces" estrategias para afrontar situaciones estresantes. Las diversas estrategias de afrontamiento posibles reevaluación positiva, búsqueda de apoyo y planificación alcanzaron promedios de $3.15 \pm 0.73,2.90 \pm 0.84$ y $3.01 \pm 0.75$, respectivamente. Las opciones (individuales) de estrategias de afrontamiento que con más frecuencia emplearon los estudiantes ante situaciones estresantes fueron principalmente las orientadas a la reevaluación positiva, le continuaron en frecuencia de las estrategias de búsqueda de apoyo social y las orientadas a la planeación (Tabla 4).

Tabla 4.

Estadística descriptiva para cada uno de los ítems de la escala de estrategias de afrontamiento al estrés académico (A-CEA), en estudiantes de una institución universitaria, Cartagena, 2018.

\begin{tabular}{|c|c|c|c|c|c|c|c|c|}
\hline \multirow[b]{2}{*}{ Item } & \multirow[b]{2}{*}{ Media } & \multirow[b]{2}{*}{$\mathrm{DE}$} & \multirow[b]{2}{*}{ Ranking } & \multicolumn{5}{|c|}{ Proporción de Respuesta (\%) } \\
\hline & & & & 1 & 2 & 3 & 4 & 5 \\
\hline Pensar que soy capaz & 3.41 & 1.09 & 1 & 2.88 & 20.70 & 27.12 & 31.02 & 18.28 \\
\hline Centrarse en lo que necesita & 3.33 & 1.05 & 2 & 3.03 & 20.49 & 31.23 & 30.20 & 15.05 \\
\hline Pensar que saldrá bien & 3.31 & 1.10 & 3 & 4.78 & 19.88 & 29.69 & 29.89 & 15.77 \\
\hline Cambiar algunas cosas & 3.30 & 1.09 & 4 & 4.11 & 22.34 & 27.58 & 31.33 & 14.64 \\
\hline Pensar en positivo & 3.26 & 1.11 & 5 & 4.52 & 23.37 & 28.40 & 28.09 & 15.61 \\
\hline Pensar objetivamente & 3.24 & 1.10 & 6 & 3.95 & 24.76 & 29.12 & 27.22 & 14.95 \\
\hline Dar un plazo para solucionar & 3.22 & 1.10 & 7 & 4.21 & 25.32 & 29.22 & 26.55 & 14.69 \\
\hline Pensar que estoy preparado & 3.10 & 1.11 & 8 & 7.14 & 24.96 & 29.02 & 27.68 & 11.20 \\
\hline Verlo como lógico y normal & 3.10 & 1.14 & 9 & 8.12 & 24.29 & 28.09 & 27.73 & 11.76 \\
\hline Priorizar tareas & 3.07 & 1.07 & 10 & 4.88 & 29.53 & 29.69 & 25.32 & 10.58 \\
\hline Expresa opiniones y busca apoyo & 3.00 & 1.07 & 11 & 4.98 & 34.05 & 25.37 & 26.66 & 8.94 \\
\hline Organizar recursos personales & 3.00 & 1.07 & 12 & 6.47 & 29.12 & 31.43 & 23.83 & 9.14 \\
\hline Hablar para documentarme & 3.00 & 1.14 & 13 & 9.19 & 27.12 & 28.76 & 24.29 & 10.63 \\
\hline Planificar como estudiar & 2.95 & 1.13 & 14 & 8.12 & 32.05 & 26.19 & 23.57 & 10.07 \\
\hline Hablar con pareja, familia, amigos & 2.95 & 1.23 & 15 & 12.17 & 29.22 & 23.52 & 21.93 & 13.15 \\
\hline Consejo a un familiar o amigo & 2.94 & 1.21 & 16 & 11.04 & 31.59 & 21.88 & 23.01 & 12.48 \\
\hline Buscar consejo y pedir ayuda & 2.90 & 1.17 & 17 & 10.73 & 32.05 & 22.75 & 24.81 & 9.66 \\
\hline No dar importancia & 2.87 & 1.10 & 18 & 10.07 & 30.77 & 28.35 & 23.47 & 7.34 \\
\hline Manifestar sentimientos & 2.86 & 1.15 & 19 & 10.89 & 31.69 & 26.81 & 21.01 & 9.60 \\
\hline Olvidar aspectos desagradables & 2.81 & 1.13 & 20 & 8.94 & 40.01 & 20.80 & 21.42 & 8.83 \\
\hline Elaborar un plan de acción & 2.72 & 1.15 & 21 & 15.05 & 31.79 & 25.63 & 20.75 & 6.78 \\
\hline Hablar los problemas con otros & 2.70 & 1.17 & 22 & 14.54 & 37.49 & 20.49 & 19.21 & 8.27 \\
\hline Hacer lista de tareas por hacer & 2.70 & 1.18 & 23 & 16.64 & 31.59 & 25.37 & 18.39 & 8.01 \\
\hline
\end{tabular}


En orden de frecuencia se encontró que los estudiantes respondieron que cuando me enfrento a una situación problemática durante los exámenes, procuro pensar que soy capaz de hacer las cosas bien por mí mismo (reevaluación positiva), me centro en lo que necesito para obtener los mejores resultados (planeación), suelo pensar que me saldrán bien (reevaluación positiva), pero también los estudiantes consideran buscar apoyo cuando se les presenta una situación que les genera problema (búsqueda de apoyo) (Tabla 4).

Los estudiantes utilizan con menos frecuencia ante una situación que les genera problema el olvidar aspectos desagradables (reevaluación positiva), elaborar un plan de acción o hacer una lista de tareas (planeación) y hablar de los problemas con otros (búsqueda de apoyo social) (Tabla 4).

Al revisar las respuestas de los estudiantes de la Facultad de Ciencias Económicas, se encontró que el $28.2 \%$ manifestó que ante una situación problemática resaltan los positivo de ella, el $26,7 \%$ no permite que el problema les impida cumplir con sus compromisos. La reevaluación positiva es el recurso de afrontamiento más utilizado ya que el 32,9\% organizan los recursos personales que tiene para afrontar dicha situación. La planeación es otro de los recursos utilizados por el 32,3\% de los estudiantes para la preparación de los exámenes y el 30.8\% utiliza el apoyo emocional como estrategia de afrontamiento.

Al asociar la frecuencia de uso de cada una de las estrategias de afrontamiento investigadas para cada situación estresante, se encontraron diferencias estadísticamente significativas para los estresores que se muestran en la Tabla 5. La mayoría de los estudiantes ante las situaciones estresantes utilizan la estrategia de afrontamiento reevaluación positiva. Es así como son estadísticamente significativas y se utiliza en orden de frecuencia la reevaluación positiva, la planificación y el apoyo social ante la competencia por las calificaciones $(p=0.00)$; de igual forma se utilizan estas estrategias ante el estresor debilidad de carácter $(p=0.00)$; profesores críticos $(p=0.00)$; cantidad de trabajo $(\mathrm{p}=0.02)$.

Ante el éxito en el futuro y ser un estudiante exitoso, los estudiantes utilizan en orden de frecuencia estrategias de afrontamiento de reevaluación positiva, de planeación y de apoyo social ( $\mathrm{p}=0.00$ ); igual uso de estrategias se presenta ante la reprobación de asignaturas ( $\mathrm{p}=0.00)$; ante las decisiones académicas los estudiantes utilizan en orden de frecuencia similares estrategias de afrontamiento $(p=0.00)$ y ante los tiempos para relajarme y tiempo académico, la frecuencia de uso de las estrategias es similar, con un resultado de $\mathrm{p}=0.00$ (Tabla 5 ). 
Tabla 5.

Asociación entre estrés académico percibido y las estrategias de afrontamiento al estrés académico utilizadas por los estudiantes de una institución universitaria, Cartagena, 2018.

\begin{tabular}{|c|c|c|c|c|c|c|c|}
\hline \multicolumn{3}{|c|}{ Reevaluación Positiva } & \multicolumn{2}{|c|}{ Búsqueda de Apoyo } & \multicolumn{2}{|c|}{ Planificación } & \multirow{2}{*}{$\begin{array}{c}\text { p- } \\
\text { Valor }\end{array}$} \\
\hline & Media & $\mathrm{DE}$ & Media & $\mathrm{DE}$ & Media & $\mathrm{DE}$ & \\
\hline \multicolumn{8}{|c|}{ 1. Competencia por calificaciones } \\
\hline No & 3.03 & 0.73 & 2.82 & 0.71 & 2.90 & 0.75 & \multirow{2}{*}{0.00} \\
\hline Sí & 3.22 & 0.71 & 2.95 & 0.70 & 3.07 & 0.74 & \\
\hline
\end{tabular}

2. Expectativas de los Padres

$\begin{array}{llllllll}\text { No } & 3.15 & 0.72 & 2.92 & 0.84 & 3.00 & 0.75 & 0.79 \\ \text { Sí } & 3.14 & 0.73 & 2.90 & 0.85 & 3.01 & 0.75 & \end{array}$

\begin{tabular}{|c|c|c|c|c|c|c|c|}
\hline \multicolumn{8}{|c|}{ 3. Período de exámenes } \\
\hline No & 3.16 & 0.77 & 2.90 & 0.86 & 3.02 & 0.76 & \multirow{2}{*}{0.84} \\
\hline Sí & 3.15 & 0.72 & 2.91 & 0.84 & 3.00 & 0.74 & \\
\hline \multicolumn{8}{|c|}{ 4. Debilidad de carácter } \\
\hline No & 3.20 & 0.77 & 2.89 & 0.87 & 3.00 & 0.76 & \multirow{2}{*}{$0.00^{*}$} \\
\hline Sí & 3.11 & 0.70 & 2.93 & 0.81 & 3.01 & 0.74 & \\
\hline
\end{tabular}

\section{Profesores críticos}

$\begin{array}{llllllll}\text { No } & 3.06 & 0.76 & 2.78 & 0.84 & 2.94 & 0.78 & 0.00^{*} \\ \text { Sí } & 3.19 & 0.71 & 2.96 & 0.84 & 3.04 & 0.73 & \end{array}$

\begin{tabular}{cccccccc}
\multicolumn{7}{c}{ 6. Cantidad de trabajo } \\
No & 3.13 & 0.75 & 2.83 & 0.84 & 2.96 & 0.77 & $0.02^{*}$ \\
Sí & 3.15 & 0.72 & 2.94 & 0.84 & 3.03 & 0.73 &
\end{tabular}

7. Tamaño del plan de estudios

$\begin{array}{llllllll}\text { No } & 3.14 & 0.74 & 2.87 & 0.84 & 2.97 & 0.75 & 0.14 \\ \text { Sí } & 3.16 & 0.72 & 2.92 & 0.84 & 3.03 & 0.75 & \end{array}$


Tabla 5 .

Asociación entre estrés académico percibido y las estrategias de afrontamiento al estrés académico utilizadas por los estudiantes de una institución universitaria, Cartagena, 2018.

\begin{tabular}{|c|c|c|c|c|c|c|c|}
\hline \multicolumn{3}{|c|}{ Reevaluación Positiva } & \multicolumn{2}{|c|}{ Búsqueda de Apoyo } & \multicolumn{2}{|c|}{ Planificación } & \multirow{2}{*}{$\begin{array}{c}\text { p- } \\
\text { Valor }\end{array}$} \\
\hline & Media & $\mathrm{DE}$ & Media & $\mathrm{DE}$ & Media & $\mathrm{DE}$ & \\
\hline \multicolumn{8}{|c|}{ 8. Conseguir trabajo } \\
\hline No & 3.16 & 0.75 & 2.91 & 0.86 & 3.00 & 0.75 & \multirow{2}{*}{0.83} \\
\hline Sí & 3.15 & 0.72 & 2.91 & 0.83 & 3.01 & 0.75 & \\
\hline
\end{tabular}

9. Preguntas de los exámenes

$\begin{array}{llllllll}\text { No } & 3.13 & 0.78 & 2.86 & 0.85 & 3.02 & 0.76 & 0.26 \\ \text { Sí } & 3.16 & 0.71 & 2.92 & 0.84 & 3.00 & 0.74 & \end{array}$

10. Éxito en el futuro

$\begin{array}{llllllll}\text { No } & 2.75 & 0.73 & 2.70 & 0.80 & 2.72 & 0.76 & 0.00^{*} \\ \text { Sí } & 3.20 & 0.71 & 2.93 & 0.84 & 3.04 & 0.74 & \end{array}$

11. Estudiante exitoso (a)

$\begin{array}{llllllll}\text { No } & 2.80 & 0.75 & 2.76 & 0.87 & 2.74 & 0.76 & 0.00^{*} \\ \text { Sí } & 3.19 & 0.72 & 2.93 & 0.81 & 3.04 & 0.74 & \end{array}$

12. Reprobar asignaturas

$\begin{array}{llllllll}\text { No } & 3.11 & 0.79 & 2.78 & 0.85 & 2.95 & 0.75 & 0.00^{*} \\ \text { Sí } & 3.16 & 0.50 & 2.95 & 0.84 & 3.03 & 0.75 & \end{array}$

13. Decisiones académicas

$\begin{array}{llllllll}\text { No } & 2.84 & 0.75 & 2.69 & 0.87 & 2.74 & 0.77 & 0.00^{*} \\ \text { Sí } & 3.22 & 0.50 & 2.96 & 0.83 & 3.07 & 0.73 & \end{array}$

14. Tiempo para relajarme

$\begin{array}{llllllll}\text { No } & 3.04 & 0.75 & 2.85 & 0.83 & 2.95 & 0.78 & 0.00^{*} \\ \text { Sí } & 3.20 & 0.71 & 2.94 & 0.81 & 3.04 & 0.73 & \end{array}$


Tabla 5.

Asociación entre estrés académico percibido y las estrategias de afrontamiento al estrés académico utilizadas por los estudiantes de una institución universitaria, Cartagena, 2018.

\begin{tabular}{|c|c|c|c|c|c|c|c|}
\hline \multicolumn{3}{|c|}{ Reevaluación Positiva } & \multicolumn{2}{|c|}{ Búsqueda de Apoyo } & \multicolumn{2}{|c|}{ Planificación } & \multirow{2}{*}{$\begin{array}{c}\text { p- } \\
\text { Valor }\end{array}$} \\
\hline & Media & $\mathrm{DE}$ & Media & $\mathrm{DE}$ & Media & $\mathrm{DE}$ & \\
\hline \multicolumn{8}{|c|}{ 15. Tiempo académico } \\
\hline No & 3.00 & 0.74 & 2.81 & 0.86 & 2.90 & 0.76 & \multirow{2}{*}{$0.00^{*}$} \\
\hline Sí & 3.20 & 0.72 & 2.94 & 0.83 & 3.05 & 0.74 & \\
\hline \multicolumn{8}{|c|}{ 16. Expectativas de Profesores } \\
\hline No & 3.16 & 0.75 & 2.90 & 0.86 & 3.02 & 0.77 & \multirow{2}{*}{0.84} \\
\hline Sí & 3.14 & 0.50 & 2.91 & 0.83 & 3.00 & 0.73 & \\
\hline \multicolumn{8}{|c|}{ 17. Tiempo de los exámenes } \\
\hline No & 3.15 & 0.75 & 2.88 & 0.87 & 3.01 & 0.76 & \multirow{2}{*}{0.71} \\
\hline Sí & 3.15 & 0.72 & 2.92 & 0.83 & 3.01 & 0.74 & \\
\hline \multicolumn{8}{|c|}{ 18. Incapaz de ponerme al día } \\
\hline No & 3.18 & 0.75 & 2.91 & 0.87 & 3.04 & 0.77 & \multirow{2}{*}{0.07} \\
\hline Sí & 3.10 & 0.70 & 2.90 & 0.80 & 2.97 & 0.72 & \\
\hline
\end{tabular}

DE: desviación estándar. *Valores de p estadísticamente significativos

\section{DISCUSIÓN}

El estrés académico que presentan los estudiantes matriculados en una institución universitaria en la modalidad presencial, está relacionado principalmente con la competencia para las calificaciones y la principal estrategia de afrontamiento utilizada es la reevaluación positiva, seguida en orden de importancia por las estrategias de planificación y búsqueda de apoyo social. (Fernández L, 2015) Expresa que las emociones relacionadas con el optimismo y el pesimismo son aspectos influyentes 
en las manifestaciones del estrés, no así la autoestima cuyos resultados no fueron significativos; recibir ayudas ante las dificultades (apoyo social instrumental) facilita el afrontamiento ante las demandas de estrés (Fernández L, 2015). El estudio de (Cabanach, 2013) "Diferencias en el afrontamiento del estrés en estudiantes universitarios hombres y mujeres" refiere que las estrategias de afrontamiento reevaluación positiva y planificación son utilizadas en mayor medida por los hombres, las mujeres utilizan con más frecuencia la estrategia de apoyo social.

Contrario a lo encontrado en el presente estudio, Cabanach R y Casari L (Cabanach, 2013) (Casari L, 2014) reportan que los estudiantes utilizan con más frecuencia como estrategia de afrontamiento la búsqueda de gratificaciones alternativas y la descarga emocional, discriminando por sexo su utilización, expresan que la reevaluación positiva es utilizada con mayor frecuencia por las mujeres y por los hombres la descarga emocional. El afrontamiento al estrés se ve facilitado por poseer un temperamento optimista o bien por tener ayudas que permiten manejar mejor la ansiedad y el estrés, por lo que se hace necesario identificar en los jóvenes el pesimismo, fortalecerlos en el optimismo pues a medida que se incrementa éste, disminuyen las manifestaciones emocionales del estrés (Fernández L, 2015), al igual que incrementar y fortalecer en los estudiantes las redes de apoyo social para permitirles tener mayores herramientas con las cuales afrontar el estrés académico. Para (Cabanach, 2010) el desarrollar estas estrategias orientadas a fortalecer las creencias de autoeficacia permite que el estudiante responda de manera adecuada a las exigencias académicas, a mejorar la motivación ante el aprendizaje y a alcanzar mayores niveles de bienestar.

La distribución por sexo de los estudiantes universitarios participantes del presente estudio es ligeramente similar, a diferencia de los datos encontrados por (Fernández L, 2015) (Cabanach, 2013), donde la población de estudiante fue primordialmente femenina, con edades similares a este estudio. A pesar de que en el presente estudio se agruparon a hombres y mujeres, los resultados coinciden a los encontrados por (Cabanach, 2013), en cuanto a las estrategias de afrontamiento utilizadas por los estudiantes y (Fernández L, 2015) anota que la manifestación de emocionalidad es mayor en las mujeres y con relación a la edad, afirma que esta puede disminuir la importancia del apoyo social instrumental, no así con el apoyo emocional.

En los estudiantes participantes, los resultados arrojaron que la autopercepción académica es uno de los factores que mayor estrés les genera representada en el éxito como su mayor preocupación, tanto en su etapa como estudiante, como alcanzarlo en el desempeño de su futura carrera.

La carga académica constituye un elemento estresor en los estudiantes participantes, (Casari L, 2014) reporta que la situación de los exámenes les genera a los estudiantes ansiedad y ante esta situación utilizan estrategias de afrontamiento de gratificaciones alternativas y descarga emocional. (Solano O, 2014) Encontró que la sobrecarga académica y la deficiencia metodológica se constituyen en estresores de los estudiantes de su estudio. El motivar al estudiante para el aprendizaje permite que ellos mejoren los niveles de bienestar con altas creencias de autoeficacia y desarrollen estrategias de afrontamiento que les permiten responder a las demandas académicas de manera eficiente (Cabanach, 2010). El estrés ocasionado por la competitividad en los estudiantes es otro factor importante ya que son limitadas las oportunidades laborales. Todos estos factores de estrés ocasionan en los estudiantes la puesta en marcha de estrategias de afrontamiento que le permitan afrontar las demandas que les presenta el medio, pero 
no todos responden de la misma manera y en ocasiones las exigencias pueden dañar su salud o adoptar estrategias inadecuadas (Solano O, 2014).

La tensión originada por el estrés es en cierta medida necesaria para el desarrollo de la personalidad del estudiante; sin embargo, cuando el estrés va más allá de los niveles óptimos agota sus energías, deteriora su desempeño y puede llegar a dañar su salud. Así mismo, lo que es considerado estresante para unos no lo es para otros, originando que los estudiantes en un intento de adaptarse a estas situaciones pongan en marcha una serie de estrategias de afrontamiento para superar con éxito las exigencias que se les demanda, buscando el alivio de su estado tensional; sin embargo, una gran parte de estudiantes universitarios carecen de estrategias o adoptan estrategias inadecuadas (Solano O, 2014).

Para los participantes del estudio la reevaluación positiva es la estrategia de afrontamiento más utilizada, al respecto Cabanach (Cabanach, 2010) reporta que la percepción de autoeficacia del estudiante es importante para afrontar de manera positiva cada uno de los estresores académicos y responder de manera adecuada al estrés y encontró una correlación significativa además con las estrategias de planificación. Es fortaleciendo estas estrategias como se acompaña al estudiante en su proceso de maduración y en lograr que alcancen a desarrollar estrategias adecuadas ante los diferentes estresores académicos que se les presentan.

\section{CONCLUSIONES}

En el presente estudio se identificó como principal estresor el éxito: como estudiante el período de exámenes y las preguntas que lo conforman se constituyen en factores de estrés y como profesionales el ejercicio de la carrera. La utilización de estrategias de afrontamiento contempla la reevaluación positiva y la planeación, siendo estas las mas empleadas o utilizadas con mayor frecuencia.

Continuar esta línea de estudio determinando por género las respuestas de afrontamiento de los estudiantes ante los diferentes estresores académicos, permitirá establecer sobre cuales elementos intervenir para alcanzar un fortalecimiento del estudiante ante las situaciones de estrés. Este tipo de intervenciones no solo estará orientada a mejorar la motivación del estudiante para el aprendizaje, sino también y en consecuencia a lograr mayores niveles de bienestar.

\section{FUENTES DE FINANCIAMIENTO}

Para desarrollar el presente estudio se contó con apoyo financiero institucional a través de Resolución 00473 de marzo 2 de 2016 y acta de compromiso Nº 0421-2017.

\section{CONSIDERACIONES ÉTICAS}

El presente estudio se acoge a lo dispuesto en la Declaración de Helsinki modificada en Edimburgo, así como también a lo estipulado en la resolución 008430 de octubre de 1993 (República de Colombia. Ministerio de Salud, 1993,) mediante la cual se estima de riesgo nulo. No obstante, se solicitó consentimiento informado escrito para cada participante. 


\section{REFERENCIAS BIBLIOGRÁFICAS}

Arrieta, K, Diaz, S, Gonzalez, F. (2013). Síntomas de depresión, ansiedad y estrés en estudiantes de odontología: prevalencia y factores relacionados. . rev. colomb. psiquiat. , 42(2), 173-181.

Beaton, D. E., Bombardier, C., Guillemin, F., \& Ferraz, M. B. (2010). Guidelines for the process of crosscultural adaptation of self-report measures. Spine, 25(4), 3186-91.

Bedewy, D., \& Gabriel, A. (2015).Examining perceptions of academic stress and its sources among university students: The Perception of Academic Stress Scale. Health Psychology Open, 2(2), 1-9. Obtenido de https://journals.sagepub.com/doi/pdf/10.1177/2055102915596714

Cabanach, R. G., Valle, A., Rodríguez, S., Piñeiro, I., \& Freire, C. (2010). Escala de afrontamiento del estrés académico (A-CEA). . Revista Iberoamericana de Psicología y Salud., 1(1), 51-64. Obtenido de https://www.redalyc.org/html/2451/245116411005/

Cabanach, R. G., Valle, A., Martínez, S. R., Piñeiro, I., \& Millán, P. G. (2010).Las creencias motivacionales como factor protector del estrés en estudiantes universitarios. Eur. j. educ. psychol., 3(1), 75-87.

Cabanach, R. G., Fariña, F., Freire, C., González, P., \& del Mar Ferradás, M. (2015). Diferencias en el afrontamiento del estrés en estudiantes universitarios hombres y mujeres. Eur. j. educ. psychol, 6(1), 19-32.

Casari, L. M., Anglada, J., \& Daher, C. (2014). Estrategias de afrontamiento y ansiedad ante exámenes en estudiantes universitarios. . Revista de Psicología., 32(2), 243-269.

Fernández, L, Gonzalez, A, \& Trianes, M. (2015). Relaciones entre estrés académico, apoyo social, optimismo-pesimismo y autoestima en estudiantes universitarios. Ejrep., 13(1), 111-130.

Fernández, M. (2009). Estrés percibido, estrategias de afrontamiento y sentido de coherencia en estudiantes de enfermería: su asociación con salud psicológica y estabilidad emocional. UNIVERSIDAD DE LEÓN : Departamento de Psicología, Sociología y Filosofía .

García, J, Redin, A, López, J. (2013). Cálculo del tamaño de la muestra en investigación en educación médica. Inv ed Med., 2(8), 217-224. Obtenido de http://riem.facmed.unam.mx/sites/all/ archivos/V2Num04/07_MIE_CA

García R., Pérez, F., Pérez-, J., \& Natividad, L. (2012). Evaluación del estrés académico en estudiantes de nueva incorporación a la universidad. Rev.latinoam.psicol. , 44(2), 143-154. Obtenido de http://www.scielo.org.co/pdf/rlps/v44n2/v44n2a01.pdf\#page=143

Gómez, L, Zavala, D, López, M., \& Sánchez, R. (2012). El estrés como un factor de riesgo en la salud: análisis diferencial entre docentes de universidades públicas y privadas. (s.f.).

González R, Fernandez, L, Gonzalez, D. (2010). Estresores académicos percibidos por estudiantes universitarios de ciencias de la salud. Fisioterapia, 32(4), 151-158. Obtenido de https://ruc.udc.es/dspace/bitstream/handle/2183/16929/GonzlaezCabanach_ Estresores.pdf?sequence=3\&isAllowed $=\mathrm{y}$

Gutiérrez, J, Montoya, L, Toro, B, Briñón, M, Rosas, E, \& Salazar, L.(2010). Depresión en estudiantes universitarios y su asociación con el estrés académico. . Rev CES Med, 24(1), 7-17.

Lazarus R. (1984). Stress, appraisal and coping. New York:. New York: Springer Publishing Company. 
Lumley, M. A., \& Provenzano, K. M. (2003). Stress management through written emotional disclosure improves academic performance among college students with physical symptoms. Journal of Educational Psychology., 95(3), 641-649.

Montalvo-Prieto, A., Blanco-Blanco, K., Cantillo-Martínez, N., Castro-González, Y., Downs-Bryan, A., \& Romero-Villadiego, E. (2015). Estrés académico en los estudiantes del área de la salud en una universidad pública, Cartagena-Colombia. . Rev.cienc.biomed. , 6(2), 309-318.

Piemontesi, S. E., Heredia, D. E., Furlan, L. A., Sánchez-Rosas, J., \& Martínez, M. (2012). Ansiedad ante los exámenes y estilos de afrontamiento. Anales de Psicología., 28(1), 89-96.

Polo, A., López, J. M. H., \& Muñoz, C. P. (1996). Evaluación del estrés académico en estudiantes universitarios. Ansiedad y estrés, 2(2), 159-172.

República de Colombia. Ministerio de Salud. Resolución 008430 de 1993, octubre 1993. Por la cual se establecen las normas científicas, técnicas y administrativas para la investigación en salud. Bogotá, Colombia. Disponible en: https://www.minsalud.gov.co/. (s.f.).

Reyes L, (2012). El estrés como un factor de riesgo en la salud: análisis diferencial entre docentes de universidades públicas y privadas. Revista unam.mx. .

Rost, D. H., \& Schermer, F. J. (2008). DAI Differentielles Leistungsangst Inventar. (Vol. 2). Handbuch.

Sandín, B., \& Chorot, P. (2003). Cuestionario de afrontamiento del Estrés (CAE): desarrollo y validación preliminar. Revista de Psicopatología y Psicol

SEAS, S. E. (2016). XII Congreso Internacional de la Sociedad Española para el Estudio de la Ansiedad y el Estrés -SEAS. Obtenido de https:// www.google.com/search?=Sociedad +Espa\%C3\%B1ola +para +el +estudio +de +la +ansiedad +y +el +estr\%C3\%A9 +SEAS\&oq=Sociedad +Espa\%C3\%B1ola +para +el testudio tde +la tansiedad +y +el +estr\%C3\%A9s +SEAS\&aqs=chrome..69i57j0.460j0j7\&sourceid=chrome\&ie=UTF-8.

Solano, Y. O., Gómez, D., Cárdenas, A., Bartolo, F., Martinez, B., Bolaños, O., \& Mendoza, J. (2016). Estudio del estrés académico en estudiantes universitarios de la Facultad de Ciencias Matemáticas utilizando métodos multivariantes. Pesquimat, 17(1).

Ticona Benavente, S. B., Paucar Quispe, G., \& Llerena Callata, G. (2010). Nivel de estrés y estrategias de aprontamiento en estudiantes de la facultad de enfermería: UNSA Arequipa. 2006. Enfermería global.19(18), 1-18. Recuperado el 12 de 2018, de http://scielo.isciii.es/pdf/eg/ n19/docencia1.pdf 